\title{
Do native and invasive herbivores have an effect on Brassica rapa pollination?
}

\author{
Scopece, G ; Frachon, Léa ; Cozzolino, Salvatore
}

\begin{abstract}
Mutualistic (e.g. pollination) and antagonistic (e.g. herbivory) plant-insect interactions shape levels of plant fitness and can have interactive effects. By using experimental plots of Brassica rapa plants infested with generalist (Mamestra brassicae) and specialised (Pieris brassicae) native herbivores and with a generalist invasive (Spodoptera littoralis) herbivore, we estimated both pollen movement among treatments and the visiting behaviour of honeybees versus other wild pollinators. Overall, we found that herbivory has weak effects on plant pollen export, either in terms of inter-treatment movements or of dispersion distance. Plants infested with the native specialised herbivore tend to export less pollen to other plants with the same treatment. Other wild pollinators preferentially visit non-infested plants that differ from those of honeybees, which showed no preferences. Honeybees and other wild pollinators also showed different behaviours on plants infested with different herbivores, with the former tending to avoid revisiting the same treatment and the latter showing no avoidance behaviour. When taking into account the whole pollinator community, i.e. the interactive effects of honeybees and other wild pollinators, we found an increased avoidance of plants infested by the native specialised herbivore and a decreased avoidance of plants infested by the invasive herbivore. Taken together, our results suggest that herbivory may have an effect on B. rapa pollination, but this effect depends on the relative abundance of honeybees and other wild pollinators.
\end{abstract}

DOI: https://doi.org/10.1111/plb.12985

Posted at the Zurich Open Repository and Archive, University of Zurich

ZORA URL: https://doi.org/10.5167/uzh-170465

Journal Article

Accepted Version

Originally published at:

Scopece, G; Frachon, Léa; Cozzolino, Salvatore (2019). Do native and invasive herbivores have an effect on Brassica rapa pollination? Plant Biology, 21(5):927-934.

DOI: https://doi.org/10.1111/plb.12985 
1 Do native and invasive herbivores have an effect on Brassica rapa pollination?

2

3 G. Scopece ${ }^{1}$, L. Frachon ${ }^{1,2}$, S. Cozzolino ${ }^{1 *}$

4

$5{ }^{1}$ Department of Biology, University of Naples Federico II, Complesso Universitario MSA, via

6 Cinthia I-80126, Naples, Italy

7

8

$9{ }^{2}$ Department of Systematic and Evolutionary Botany, University of Zurich, Zollikerstrasse 107, 10 8008, Zurich, Switzerland

11

12 Running title: Herbivory and pollination in Brassica rapa

13

$14 \quad{ }^{*}$ Corresponding author: Salvatore Cozzolino, cozzolin@unina.it, +38081679185.

15

16 Keywords: Herbivory; Honey bees; Mamestra brassicae; Pieris brassicae; Spodoptera littoralis; 17 wild pollinators.

18 
Abstract

- Mutualistic (e.g. pollination) and antagonistic (e.g. herbivory) plant-insect interactions shape levels of plant fitness and can have interactive effects.

- By using experimental plots of Brassica rapa plants infested with generalist (Mamestra brassicae) and specialized (Pieris brassicae) native herbivores and with a generalist invasive (Spodoptera littoralis) herbivore, we estimated both pollen movement among treatments and the visiting behaviour of honey bees versus other wild pollinators.

- Overall, we found that herbivory has weak effects on plant pollen export either in terms of inter-treatment movements or of dispersion distance. Plants infested with the native specialized herbivore tend to export less pollen on other plants with the same treatment. Other wild pollinators preferentially visit non-infested plants differently from honey bees that showed no preferences. Honey bees and other wild pollinators also showed different behaviours facing plants infested by different herbivores with the former tending to avoid revisiting the same treatment and the latter showing no avoidance behaviour. When taking into account the whole pollinator community, i.e. the interactive effects of honey bees and

- Taken together, our results suggest that herbivory may have an effect on $B$. rapa pollination but this effect depends on the relative abundance of honey bees and other wild pollinators. 


\section{INTRODUCTION}

Plant-insect interactions are among the main factors that shape plant fitness and, consequently, crop yields (e.g. Schemske \& Bradshaw 1999; Cornell and Hawkins 2003; Garibaldi et al. 2011; Rosas-Guerrero et al. 2014; Gòmez et al. 2015; Stein et al. 2017). These interactions can be either herbivores can have direct or indirect detrimental effects on plant fitness. Herbivores can affect the number of flowers produced (Litto et al. 2015) and decrease the plant resources allocable in seed production directly influencing plant fitness (Strauss et al. 1996; Mothershead \& Marquis 2000; Knauer \& Schiestl 2017) or can change the attractiveness of plants to pollinators indirectly influencing plant fitness.

Plants communicate with insects by producing compounds that can be either attractive or repellent depending on the antagonistic-mutualistic nature of the interaction (Schiestl \& Johnson 2013). From an evolutionary perspective, natural selection is expected to keep the communication channels of these interactions separate because plants should produce both compounds attractive to pollinators (but not for herbivores) and repellent for herbivores (but not for pollinators) (Parachnowitsch et al. 2012; Knauer \& Schiestl 2017). Nonetheless, in the face of herbivore attacks, plants may respond by changing floral volatile production, floral display or nectar production, which is likely to affect pollinator attractiveness (Herrera et al. 2002; Knauer \& Schiestl 2017; Glaum \& Kessler 2017). This effect can be positive or detrimental for plant fitness, i.e. it can determine a higher or a lower attractiveness to pollinators (e.g. Cozzolino et al. 2015; Litto et al. 2015; Knauer \& Schiestl 2017). Although several studies investigated the interactive effect of pollinators and herbivores on plant fitness (Galen \& Cuba 2001; Gòmez 2003, 2005, 2008; Cariveau et al. 2004; Rey et al. 2006; Parachnowitsch \& Caruso 2008; Wise \& Hebert 2010; Sletvold et al. 2015; Sauve et al. 2016), herbivory impact on pollinator foraging preferences and inter-individual gene flow within plant patches are little investigated. 
69 Under herbivore attack, the subsequently induced volatiles can affect plant pollination both at

70 individual and population level (Liao et al. 2013). Plant populations subject to herbivore attack may

71 not only experience a reduction in individual pollination success, but also an alteration in the

72 pattern of pollinator-mediated pollen transfer within and among plant patches. In the presence of induced volatiles, pollinators can change their foraging preference affecting both the pollination success of healthy plants and pollen flow between attacked and healthy plants (Krupnick et al. 1999). Despite most entomophilous plant species employing highly generalist pollination strategies responses to pollinators focused on a single insect species (e.g. Knauer \& Schiestl 2017). Yet, it is important to understand the joint effect of the entire pollinator community and the contribution of each pollinator group (but see Rusman et al. 2018). For instance, by displaying different behaviours, the relative abundance of honey bees and wild pollinators in a population can alter local plant performances (Mallinger \& Gratton 2015). Due to strong concerns that have emerged of wild pollinators and honey bees have been raised worldwide (Potts et al. 2010; Rader et al. 2016), potentially threatening yields of economically important crops (Klein et al. 2007; Aizen et al. 2008; Winfree et al. 2008). The role and the behaviour of honey bees versus other wild pollinator services have been highlighted, particularly due to the involvement of wild pollinators in "pollination insurance' for wild and crop species (Winfree et al. 2007; Rader et al. 2016). Coupled with this pollinator decline, a worldwide spread of alien herbivores species is observed due to an increase in exchanges among geographically distant areas (Ward \& Masters 2007). Whilst plant species are generally well adapted to local herbivores, the appearance of alien herbivores can have important consequences on plant fitness. Indeed, evolutionary naiveté may cause plants to be less defended against invasive species or show maladaptive responses to damage, which may affect chemical signalling and interactions with pollinators (Parker et al. 2006; Gutbrodt et al. 2012; Desurmont et al. 2014). 
Brassica rapa is a generalist plant species that was reported to attract a wide spectrum of pollinator insects (Atmowidi et al. 2007; Rader et al. 2009). This species is typically infested by specialized insects, including caterpillars of the genus Pieris (Pieridae, Lepidoptera), but has also been reported to be edible for some generalist herbivore species as Mamestra brassicae

101 (Noctuidae, Lepidoptera) and alien-invasive species as the generalist Spodoptera littoralis

102 (Noctuidae, Lepidoptera) (Amin \& Gergis 2006).

103 In this study, by using experimental approaches with plants infested by generalist and specialised 104 local herbivores and by a generalist invasive herbivore, we estimated the cross effect of native and 105 alien herbivores on the foraging behaviour of pollinators in B. rapa plants and their potential impact 106 on plant pollen export. We specifically addressed the following questions:

107 i) Is pollen movement within B. rapa patches affected by the presence of native (either $108 \quad$ generalist or specialized) and alien herbivores?

109 ii) Does the local pollinator community behave differently towards plants infested by native 110 or alien herbivores? In particular, do honey bees and other wild pollinators differ in their behaviour and visiting preferences? 
Study organisms

Brassica rapa is a non-autogamous self-incompatible, annual or biennial plant species native to Eurasia (Watanabe et al. 2000). This species is widely cultivated worldwide for oilseed production and as food crops. In pollinator surveys carried out in New Zealand and Java (Indonesia), B. rapa showed a generalist pollination system with a wide variety of pollinators belonging to the orders Hymenoptera, Diptera, Lepidoptera and Coleoptera (Atmowidi et al. 2007; Rader et al. 2009). Brassica rapa is commonly attacked by Pieris brassicae caterpillars, a native specialist herbivore, whose caterpillars are specialized in feeding on the Brassicaceae family and have adapted to overcome the glucosinolate defense system, typical of this plant family (Smallegange et al. 2007). Interestingly, butterflies from the genus Pieris also act as pollinator (e.g. Rader et al. 2009). In natural populations, Brassica rapa is also attacked by generalist herbivores such as the native herbivore Mamestra brassicae, or the alien herbivore Spodoptera littoralis, a polyphagous moth, native to Egypt. S. littoralis is considered a major pest for a wide variety of host plants (Amin \& Gergis 2006) and since the 1950's it has spread around the Mediterranean basin causing serious damage to crops (CABI Invasive Species Compedium: https://www.cabi.org/isc/datasheet/51070).

130 This herbivore was also reported to be able to feed on B. rapa leaves (Schiestl et al. 2014;

131 Chabaane et al. 2015).

\section{Study area and biological material}

134 All experiments were conducted in a meadow of the Naples University Campus of Monte Sant'Angelo (hereafter referred to as MSA). This meadow is at the edge of a densely inhabited area and can be considered a disturbed habitat. Brassica rapa seeds were collected in a seminatural population from Roccamonfina (population size over 1000 individuals, Southern Italy).

138 Seed germination and plant growth were carried out in pots under standardized light (L16:D8), soil

139 and watering conditions in the greenhouse of the Biology Department at MSA.

140 Eggs from $P$. brassicae, M. brassicae and S. littoralis were obtained in different ways. For $P$. 
141 brassicae, female butterflies were caught with insect nets in the MSA Campus, placed in net cages 142 and fed with a sugar solution. B. rapa plant was inserted in the cage to serve as an egg deposition 143 site. For M. brassicae and S. littoralis, lines of the individuals used in our experiments, instead, 144 originated from populations reared at the Swedish University of Agricultural Science (Alnarp, 145 Sweden) and refreshed with new wild-collected individuals approximately every 6 months. $M$. brassicae and S. littoralis eggs were placed in a climate chamber at $22{ }^{\circ} \mathrm{C}, 60 \%$ relative humidity and L18:D6 photoperiod in the Department of Biology at MSA. After hatching, first instars were reared on an artificial diet (Elzinga et al. 2003) and the second instars (after 3-4 days) were fed with young leaves of $B$. rapa.

Herbivory application

Brassica rapa individuals were exposed to different treatments: infested (i) by Pieris brassicae, (ii) by Mamestra brassicae, (iii) by Spodoptera littoralis or (iv) not infested, as control treatment (hereafter referred to as $\mathrm{P}$-infested, M-infested, S-infested and control plants, respectively). To apply herbivory on B. rapa individuals, we used the approach described in Litto et al. (2015). Briefly, feeding was limited to a single leaf by containing caterpillars in clip cages made of Petri dishes at the beginning of the bolting stage. Feeding was monitored regularly, and the caterpillars were replaced, if necessary. In the control group, empty clip cages were applied as the control treatment. P-infested, M-infested and S-infested plants were infested with two to six caterpillars 160 (depending on larval instar), five days before flowering (i.e. when the flowering shoot was c. $10 \mathrm{~cm}$ 161 long). Damage imposed by herbivorous feeding was estimated from each plant of the three

162 herbivory treatments in 2015, taking a picture of damaged leaves with graph paper as reference. 163 Before photographing, leaves were pressed with a transparent slide to avoid three-dimensionality. Leaf damage was then measured using ImageJ software.

Experimental design

167 To estimate the effect of native ( $P$. brassicae and M. brassicae) vs alien (S. littoralis) herbivores on pollen movement mediated by local pollinator communities, we conducted two different 
experiments. The first experiment (hereafter referred to as pollen staining plots) was conducted in

170 2015. We established seven experimental plots with 20 plants, i.e. five individuals for each

171 herbivore treatment (P-infested, M-infested, and S-infested) and five control plants. For each

172 treatment, pollen from one plant was stained using a fluorescent dye. We used Orange, Blue,

173 Green and Pink dyes, alternating colours for the different treatments in the different plots to avoid a

174 "colour effect" (Van Rossum et al. 2011). In each plot, the position of plants was randomized. To

175 avoid pollination, plants used in this experiment were kept under a net in the greenhouse until

176 being used for experimental plots. Plots were exposed separately to local pollinators in the

177 meadow at MSA for two to six hours each (see Table S1 for details). We spaced B. rapa plants

178 within the plots of $0.5 \mathrm{~m}$ to avoid pollen being transferred through physical contact between

179 neighbouring plants. After exposure, plants were moved into a dark room and observed under UV-

180 light. We noted the plants that were visited by insects carrying stained pollen and the maximum

181 and average distance of stained pollen transferred among plants within each plot. Flight distance

182 within a plot can range from 0.5 (i.e. minimum distance between two plants) to $1.92 \mathrm{~m}$ (i.e.

183 maximum distance between two plants).

184 To disentangle the role and the foraging preferences of honey bees and other pollinators facing 185 different treatments, we conducted a second experiment (hereafter referred to as pollinator choice 186 plots) in spring 2017 where we directly observed the behaviour of each pollinator. Since $M$.

187 brassicae imposed significantly more damage to plants (Fig. S1), impeding a comparison with the 188 effects of the two other herbivores, we focussed on the native specialist vs invasive herbivores (i.e. 189 P-infested vs S-infested and control plants treatments). Four plots were built with 15 plants 190 randomly placed, i.e. five plants per treatment. For each plot, 5 days after the beginning of the 191 herbivory application, individuals were transferred from the greenhouse to the meadow. This 192 experiment was realized on different dates over a period of approximately three weeks to cover the 193 seasonal pollinator spectrum (May 19, May 29, June 1, June 8). Before exposure to pollinators, 194 phenotypic measures were taken from each plant. We measured the following floral traits: petal 195 length, petal width, sepal length, and sepal width. We also estimated plant maximum height and 196 the total number of open flowers, allowing to make a comparison between P-infested and S- 
197 infested plants. In this experiment, it was not possible to standardize the plant age of control plants

198 and infested individuals. Indeed, whilst infested plants were in the same phenological stage (due to 199 herbivore application 5 days before the beginning of the flowering), control plants were chosen 200 among the plants flowering in the greenhouse. Plots were then exposed to pollinators during three 201 hours in the meadow at MSA and direct observations were realized for each of the four plots. As previously, plants were placed at a distance of $0.5 \mathrm{~m}$ to avoid physical contact. All the pollinators 203 foraging the flowers were individually observed (determination, count, sequence of all the visited 204 plants) walking in the plot for three hours. Each insect that could not be directly recognized was collected and stored in ethanol for subsequent identification. We tallied the number of visits and calculated the visitation rate for each treatment for both insect groups, i.e. honey bees and other wild pollinators, as the ratio between number of visits and total number of visits.

Data analysis

210 To understand the impact of different herbivores on B. rapa, we estimated leaf damage from each 211 herbivore treatment (P-infested, M-infested and S-infested) in 2015 and tested the difference 212 between them using the Kruskall-Wallis test and a Dunn Kruskal-Wallis multiple comparison with 213 p-value adjust with Bonferroni method (R package FSA) for pairwise comparisons.

214 For the pollen staining plots, we calculated the probability of pollen from a given treatment to be 215 exported onto plants exposed to the same or different treatments. For instance, the probability of 216 pollen from a P-infested plant being exported onto another P-infested plant was calculated for each 217 plot as the number of P-infested plants receiving P-infested pollen relative to the total number of 218 plants receiving P-infested pollen. To test whether the herbivory treatment impacts on pollen 219 export, we then compared the probability of pollen export and the average distance of stained 220 pollen movement within each plot for each treatment using the Kruskal-Wallis test and a Dunn 221 Kruskal-Wallis multiple comparison with $p$-value adjust with Bonferroni method ( $\mathrm{R}$ package FSA) 222 for pairwise comparisons.

223 For the pollinator choice plots, we observed the impact of the herbivory on plant phenotypic traits 224 by comparing phenotypic traits among treatments with a Kruskal-Wallis test. To test the effect of 
225 different traits on pollinator visitation rates, we correlated phenotypic traits that showed significant

226 differences between treatments with the number of visits using the Spearman's method. To

227 understand if herbivore treatments influence honey bees or other wild pollinator visitation rates, we

228 compared the number of visits to each treatment between honey bees and other wild pollinators

229 using the Wilcoxon test and performed a Chi Square test. Finally, we tested whether the herbivore

230 treatment influences the choice of honey bees or other wild pollinators to return preferentially to a

231 plant with the same treatment or to another plant, using the Wilcoxon test.

232 All the analyses were performed using R environment (Version 1.1.423 - (c) 2009-2018 Rstudio, 233 Inc.). 


\section{RESULTS}

Herbivory damage

Whilst the leaf damage imposed by Mamestra brassicae was higher than that caused by other herbivores (Kruskal-Wallis, Chi Square 13.53; DF $=2 ; P=0.001$ ), we found no significant differences in herbivory damage imposed by the native Pieris brassicae and the invasive Spodoptera littoralis $\left(\mathrm{Z}=1.37, P_{\mathrm{adj}}=0.51 ;\right.$ Fig. S1 $)$.

Pollen staining plots

No significant differences in the probability of pollen export were observed among control plants, P-infested, M-infested and S-infested plants (Chi Square 14.77, $\mathrm{Df}=15, P=0.45$; Fig. 1a). For a given treatment, no significant differences were observed between the probability of revisiting the same plant treatment or to visit a different one, despite a non-significant trend for P-infested plants that are less likely to export pollen on other P-infested plants (control plants; Chi square $=1.44$, $\mathrm{Df}=3, P=0.70, \mathrm{P}$-infested plants; Chi square $=6.30, \mathrm{Df}=3, P=0.1, \mathrm{M}$-infested plants; Chi square $=4.72, \mathrm{Df}=3, P=0.19$, and $\mathrm{S}$-infested plants; Chi square=1.70, $\mathrm{Df}=3, P=0.64 ;$ Fig. $1 \mathrm{~b}$ ). The average flight distance for all treatments was $1.16 \mathrm{~m}(\min =0.5$, median $=1.16, \max =1.92)$ and was similar for each treatment (control plants mean=1.20m; P-infested mean=1.07m; M-infested mean $=1.13 \mathrm{~m}$; S-infested mean=1.24m). The different treatments showed no significant differences in terms of pollen dispersal range (Chi Square 4.34; $D f=3 ; P=0.23$; Fig. 1c).

Pollinator choice plots

In the four pollinator-choice plots we individually observed 104 pollinators of $B$. rapa belonging to 21 taxa. The most abundant pollinators were honey bees (Apis mellifera, $\mathrm{n}=33$, i.e. $31.7 \%$ of the total number of insect pollinators; Fig. S2). Excluding honey bees, and only considering other wild pollinators, Hymenoptera were the main pollinators ( $n=47$ out of $71,66.2 \%$ ), but the proportion of 260 Diptera ( $n=11$ out of $71,15.5 \%)$, Coleoptera $(n=7$ out of $71,9.9 \%)$ and Lepidoptera $(n=6$ out of $26171,8.5 \%$ ) was not negligible (Fig. S2). Honey bees visited on average 3.21 plants (min=1, 262 median=2, $\max =21$ ) whilst other Hymenoptera 3.20 plants ( $\min =1$, median=1, $\max =39$ ), Diptera 
2.72 plants $(\min =1$, median $=1, \max =7)$, Coleoptera 1.14 plants $(\min =1$, median $=1, \max =2)$,

264 Lepidoptera 2.83 plants ( $\min =1$, median=2, $\max =8$ ). For further comparisons, we grouped wild Hymenoptera, Diptera, Coleoptera and Lepidoptera as opposite to the honey bees (Apis mellifera). Floral traits showed no significant differences among treatments (i.e. control plant, P-infested and S-infested; Petal width: Chi square $=0.24, \mathrm{DF}=2, P=0.887$; Petal length: Chi square $=0.76$, DF $=2, P=0.683 ;$ Sepal width: Chi square $=0.37$, DF $=2, P=0.831 ;$ Sepal length: Chi Square $=$ 3.71, $\mathrm{DF}=2, P=0.156$; Fig. S3). S-infested plants were higher than $\mathrm{P}$-infested plants but not significantly (mean height of P-infested plants $=68.1 \mathrm{~mm}$; mean height of S-infested plants $=58.2$ $\mathrm{mm}$; Fig. S4) and the two herbivore treatments showed no differences in terms of number of open flowers (mean number of open flowers of P-infested plant $=61.2$; mean number of open flowers of S-infested plant = 63.9; Fig. S4). Comparison tests showed significant differences in the height of control plants with respect to $S$-infested plants (control vs $S$-infested; $Z=4.04, P_{\text {adj }}=0.0002$, Fig. S4), and in the number of open flowers between control and both herbivory treatments (control vs P-infested plant $Z=3.41, P_{\mathrm{adj}}=0.0019$, control vs $\mathrm{S}$-infested plant $\mathrm{Z}=3.00, P_{\mathrm{adj}}=0.0082$, Fig. S4). The number of open flowers is significantly correlated with the number of pollinator visits. This correlation was negative for honey bees $(\rho=-0.29, P=0.0317)$ and positive for other wild pollinators ( $\rho=0.45, P=0.0006)$. Within different treatments, we found significant correlations only in control plants for other wild pollinators (honey bees: $\rho=-0.36, P=0.1146$, other wild pollinators: $\rho=0.64$, $P=0.002$, Fig. 2a and 2b). Correlations were instead non-significant in P-infested and S-infested treatments for both honey bees and other wild pollinators (Fig. 2a and 2b). All the plant treatments attracted the same pollinator set (including Hymenoptera, Diptera, Coleoptera and Lepidoptera). Honey bees showed no preference for different treatments (35 visits on control plants, 34 on Pinfested plants, 35 on S-infested plants; Chi square=0.02, $D f=2, P=0.9903$; Fig. 3a), whilst other wild pollinators tend to visit preferentially, but not significantly, control plants ( 80 control, $65 \mathrm{P}$ infested and $56 \mathrm{~S}$-infested plants; Chi square=4.39, $\mathrm{Df}=2, P=0.112$; Fig. 3a). Honey bees and other wild pollinators also showed non-significant differences in terms of choice of the first plant in the plot (honey bees; Chi square=1.64, $\mathrm{Df}=2, P=0.44$, other wild pollinators; Chi square=2.82, $\mathrm{Df}=2, P=0.24$, Fig. $2 b$ ). Overall, in terms of visits (first one or during all the experiment), we found 
291 that other wild pollinators contributed more than honey bees, performing significantly more visits to

292 control and $\mathrm{P}$-infested treatments (all treatments: $\mathrm{W}=842.5, P=0.00004$, control: $\mathrm{W}=98, P=$

293 0.00513, P-infested: $\mathrm{W}=74.5, P=0.01405$; S-infested: $\mathrm{W}=111.5, P=0.1042$, Fig. $2 \mathrm{c}$ ).

294 At pollinator community level, after visiting a control plant, there was an equal probability of visiting 295 any treatment $(\mathrm{W}=4983, P=0.1373)$. When separating honey bees from other wild pollinators, 296 after visiting a control plant, the honey bees significantly prefer visiting a different plant treatment to 297 revisiting another control plant $(\mathrm{W}=430.5, P=0.035$, Fig. 3c). This trend was not observed for other 298 wild pollinators $(\mathrm{W}=2471, P=0.7551$, Fig. 3c). At pollinator community level, after visiting a $P$ 299 infested plants there was a lower probability of visiting another P-infested plant than another 300 treatment $(W=4809, P=0.02195)$; however, this pattern was not significant when analysing 301 honey bees and other wild pollinators independently (honey bees; $W=459, P=0.1155$, other wild 302 pollinators; $W=2296, P=0.09588$, Fig. 3c).

303 At pollinator community level, after visiting a S-infested plant there was a lower probability of 304 visiting another $\mathrm{S}$-infested plant than another treatment $(\mathrm{W}=4419.5, P=0.00062)$; this pattern 305 was significant for honey bees $(\mathrm{W}=358.5, P=0.00181)$ but not for other wild pollinators $(\mathrm{W}=$ $306 \quad 2238.5, P=0.0574$; Fig. 3c).

\section{DISCUSSION}

309 Whilst pollinator attraction and herbivore repellence may be strongly interconnected due to common signals (or common metabolic pathways) used by plants to communicate with mutualistic 311 and antagonistic insects, most studies investigated them separately (Desurmont et al. 2014). Here 312 we tested the effect of both native and alien herbivores on the local pollinator community of the 313 generalist species Brassica rapa and disentangled the role of honey bees versus other wild 314 pollinators in plant pollen movements.

315 By staining the pollen of $B$. rapa plants infested by different herbivores (native specialized, native generalist or alien), we determined pollinator movement among treatments and observed the fine-

317 scale pollen dispersal range. We found that herbivore identity does not alter intra and inter-

318 treatment pollen movements nor pollen dispersion distance. Pollen collected from a plant exposed 
319 to herbivore treatment had similar chances of reaching a plant infested by Pieris brassicae,

320 Mamestra brassicae, Spodoptera littoralis or a control plant. The only exception was observed for

321 P-infested plants that appeared to export less pollen to other P-infested plants (Fig. 1). This pattern

322 was marginally significant but likely suggests an avoidance behaviour of the pollinator community

323 of plants infested by herbivores as already reported in Kessler et al. (2011) and in Schiestl et al.

324 (2014). Control and treated plants showed no significant differences in terms of pollen dispersal

325 range (Fig. 1). However, as the maximum distance between plants within plot was less than two

326 meters, this finding is more indicative of fine-scale insect choices between different herbivore

327 treatments than of pollen transfer among distant plant patches.

328 In generalist species such as $B$. rapa, the overall pollination can be shaped by different

329 contributions from different pollinators. We found that even in a disturbed and fragmented habitat

330 as MSA, B. rapa attracts a noteworthy diversity of pollinators (Fig. S2). Overall, concordantly with

331 the previous findings (Atmowidi et al. 2007; Rader et al. 2009), honey bees were the most common

332 pollinators (31.7\%). Among other wild pollinators, Hymenoptera accounted for the highest

333 contribution, even if Diptera, Coleoptera and Lepidoptera were also responsible for a significant

334 proportion of pollen export. All plant treatments attracted all the pollinator groups, though honey

335 bees and other wild pollinators showed different preferences in response to different plant

336 treatments (i.e. control plants, P-infested and S-infested plants). Indeed, other wild pollinators

337 visited comparatively more control plants, though not significantly, whilst honey bees showed no

338 preferences and visited any treatment with the same frequency (Fig. 3). An increased

339 attractiveness of control plants to pollinators was already observed in previous studies where

340 bumble bees forage preferentially uninfested $B$. rapa plants likely mediated by visual and

341 especially olfactory floral signals (Schiestl et al. 2014; Kellenberg et al. 2016). However, these

342 studies were conducted in an experimental setup including only one pollinator type (i.e. bumble

343 bees), despite the fact that $B$. rapa is a generalist species. Our results suggest that though wild

344 pollinators can perceive the signals produced by infested plants, preferring control individuals,

345 honey bees do not rely on the same signals when foraging. Altogether, the combined action of

346 honey bees and other wild pollinators is likely to mitigate the preferential behaviour of wild 
347 pollinators, hence leading to slight differences in pollen export, in movement between infested and 348 control plants and probably in plant seed set, as also found in Brassica nigra by Rusman et al. 349 (2018).

350 Whilst it was possible to estimate the overall movement of pollen in the pollen staining plots, the 351 pollinator choice plots allowed the observation of the shifts of pollinators between plants by tracing 352 pollinator foraging behaviour after the first visited treatment. Even for this behaviour, we found 353 differences between honey bees and other wild pollinators with the former tending to avoid 354 revisiting the same treatment and the latter showing no avoidance behaviour (Fig. 3). This finding 355 suggests that the effects of herbivory on plant pollination in a generalist species as $B$. rapa can 356 depend on the composition of the pollinator community. By analysing the data at community level 357 (i.e. honey bees and other wild pollinators together) we found a lower probability of P-infested 358 plants to export pollen on other P-infested plants thus confirming the lower number of events of 359 pollen movement among P-infested plants observed in the pollen staining experiment. 360 Interestingly, this pattern is not significant when analysing honey bees and other wild pollinators, 361 independently suggesting that it can be due to an additive effect of non-significant trends.

362 Differently from what we observed in the pollen staining experiment, in the pollinator choice plots 363 we found significant avoidance behaviour after visiting a S-infested plant. This finding was due to 364 honey bees avoiding other S-infested plants and preferentially visiting control and P-infested 365 plants. This behaviour was likely masked, in the pollen staining experiment, by the cumulative 366 effect of different foraging behaviour of honey bees and other wild pollinators. Such detected 367 differences in foraging behaviour between honey bees and other wild pollinators have been widely 368 reported in literature (e.g. MacKenzie 1994) and may be due to different "optimal foraging" 369 strategies (sensu MacArthur \& Pianka 1966; Charnov 1976). Furthermore, honey bees and other 370 wild pollinators may influence each other's foraging behaviours due to competition for floral 371 resources (Shavit et al. 2009).

372 In the pollinator choice experiment, we found significant differences in floral display among 373 different treatments (either in terms of plant height and of flower number; Fig. S4). Changes of 374 flower display can be due to a resource allocation strategy following the herbivore attack (Elmqvist 
375 \& Gardfjell 1988; Quesada et al. 1995; Mutikainen \& Delph 1996) and, in principle, can affect 376 pollinator visiting behaviours (Schiestl et al. 2014; Cozzolino et al. 2015). In our experimental setup we were unable to detect whether such differences were consequence of the herbivore attack or of a non-standardized plant age of control plants (randomly chosen among available flowering control plants). Nevertheless, to detect the effect of flower display on visiting behaviour we calculated the correlation between number of visits and number of open flowers for honey bees and for other wild 381 pollinators, separately. We found a significant positive correlation between number of visits and 382 number of open flowers for other wild pollinators and a not significant negative correlation for 383 honey bees (Fig. 2). This finding suggests that other wild pollinators, differently from honey bees, 384 can select the plant to visit also depending on flower number. However, at individual level, other wild pollinators do not show selective preference for higher flower number of the first visited plant (Fig. 3, Fig. S4) and the positive correlation between number of visits and number of open flowers 387 for other wild pollinators was only significant for control plants (Fig. 2). This evidence suggests 388 that, in our experimental setting, number of open flowers is not likely to be an important factor in 389 shaping wild pollinator behaviour. This is also supported by previous experiments demonstrating a 390 predominant role of floral volatile compounds in affecting post-herbivory attractiveness of $B$. rapa 391 (Schiestl et al. 2014).

392 Even if, with the present experimental design, we were unable to identify the visual or olfactory 393 plant traits conditioning the different pollinator behaviours, taken together our results suggest that 394 the different proportion of honey bees and other wild pollinators can lead to different selective 395 pressures and call for future studies on B. rapa (or other generalist plant species) pollination by 396 manipulating relative proportions of honey bees and other wild pollinators. Furthermore, the three 397 herbivores used in our study have different ecological and behavioural characteristics beyond their 398 origin and history of coexistence with B. rapa. Thus, future studies, should preferentially also employ pairs of phylogenetically-related native and invasive herbivores, to dissect whether the 400 effects induced by herbivores on pollination can be due to their origin or to any other ecological 401 difference. 


\section{ACKNOWLEDGMENTS}

404 Authors thank Swedish University of Agricultural Science (Alnarp, Sweden) for providing Mamestra brassicae and Spodoptera littoralis eggs; Mary Longrigg and Emily Scopece for language revision; Maria Litto and Martina Campolo for help during fieldwork. Florian Schiestl, Karl Duffy and two anonymous reviewers provided very helpful comments on an earlier version of the MS. This research was carried out in the frame of Programme STAR, financially supported by UniNA.

\section{LITERATURE CITED}

Aizen M.A., Garibaldi L.A., Cunningham S.A., Klein A.M. (2008) Long-term global trends in crop yield and production reveal no current pollination shortage but increasing pollinator dependency. Current Biology 18:1572-1575.

Amin A.A., Gergis M. F. (2006). Integrated management strategies for control of cotton key pests in middle Egypt. Agronomic Research 4:121-128.

Atmowidi T., Buchori D., Manuwoto S., Suryobroto B., Hidayat P. (2007) Diversity of pollinator insects in relation to seed set of mustard (Brassica rapa L.: Cruciferae). HAYATI Journal of Biosciences 14:155-161.

Cariveau D., Irwin R.E., Brody A.K., Garcia-Mayeya L.S., von der Ohe A. (2004) Direct and indirect effects of pollinators and seed predators to selection on plant and floral traits. Oikos 104:15-26.

Chabaane Y., Laplanche D., Turlings T.C.J., Desurmont G.A. (2015) Impact of exotic insect herbivores on native tritrophic interactions: a case study of the African cotton leafworm, Spodoptera littoralis and insects associated with the field mustard Brassica rapa. Journal of Ecology 103:109-117.

Charnov E.L. (1976) Optimal foraging, the marginal value theorem. Theoretical Population Biology 9:129-36. 
Cornell H.V., Hawkins B.A. (2003) Herbivore responses to plant secondary compounds: a test of phyto- chemical coevolution theory. American Naturalist 161:507-522.

Cozzolino S., Fineschi S., Litto M., Scopece G., Trunschke J., Schiestl F.P. (2015) Herbivory increases fruit set in Silene latifolia: a consequence of induced pollinator-attracting floral volatiles? Journal of Chemical Ecology 41:622-630.

Desurmont G.A., Harvey J., van Dam N.M., Cristescu S.M., Schiestl F.P., Cozzolino S., et al. (2014) Alien interference: disruption of infochemical networks by invasive insect herbivores. Plant Cell \& Environment 37:1854-1865.

Elmqvist T., Gardfjell H. (1988) Differences in response to defoliation between males and females of Silene dioica. Oecologia 77:225-230.

Elzinga, J. A., Harvey, J. A., \& Biere, A. (2003). The effects of host weight at parasitism on fitness correlates of the gregarious koinobiont parasitoid Microplitis tristis and consequences for food consumption by its host, Hadena bicruris. Entomologia Experimentalis et Applicata 108:95-106

Galen C, Cuba J (2001) Down the tube: pollinators, predators, and the evolution of flower shape in the alpine skypilot, Polemonium viscosum. Evolution 55:1963-1971.

Garibaldi L.A., Aizen M.A., Klein A.M., Cunningham S.A., Harder L.D. (2011) Global growth and stability of agricultural yield decrease with pollinator dependence. Proceedings of the National Academy of Science USA 108:5909-5914.

Glaum P., Kessler A. (2017) Functional reduction in pollination through herbivore-induced pollinator limitation and its potential in mutualist communities. Nature communications 8:2031.

Gòmez J.M. (2003) Herbivory reduces the strength of pollinator-mediated selection in the Mediterranean herb Erysimum mediohispanicum: consequences for plant specialization. American Naturalist 162:242-256.

Gòmez J.M. (2005) Non-additive effects of herbivores and pollinators on Erysimum mediohispanicum (Cruciferae) fitness. Oecologia 143:412-418. 
Gòmez J.M. (2008) Sequential conflicting selection due to multispecific interactions triggers evolutionary trade-offs in a monocarpic herb. Evolution 62:668-679.

Gòmez J.M., Perfectti F., Lorite J. (2015) The role of pollinators in floral diversification in a clade of generalist flowers. Evolution 69:863-878.

Gutbrodt B., Dorn S., Unsicker S.B., Mody K. (2012) Species-specific responses of herbivores to within-plant and environmentally mediated between-plant variability in plant chemistry. Chemoecology 22:101-111.

Hallmann C.A., Sorg M., Jongejans E., Siepel H., Hofland N., Schwan H. et al. (2017) More than 75 percent decline over 27 years in total flying insect biomass in protected areas. PLoS One 12:e0185809.

Herrera C.M., Medrano M., Rey P.J., Sánchez-Lafuente A.M., García M.B., Guitián J., Manzaneda, A.J. (2002) Interaction of pollinators and herbivores on plant fitness suggests a pathway for correlated evolution of mutualism-and antagonism-related traits. Proceedings of the National Academy of Sciences USA 99:16823-16828.

Johnson S.D., Steiner K.E. (2000) Generalization versus specialization in plant pollination systems. Trends in Ecology \& Evolution 15:140-143.

Kellenberger, R. T., Schlüter, P. M., Schiestl, F. P. (2016) Herbivore-induced DNA demethylation changes floral signalling and attractiveness to pollinators in Brassica rapa. PloS One 11:e0166646.

Kessler, A., Halitschke, R., Poveda, K. (2011) Herbivory-mediated pollinator limitation: negative impacts of induced volatiles on plant-pollinator interactions. Ecology 92:1769-1780.

Klein A.M., Vaissiere B.E., Cane J.H., Steffan-Dewenter I., Cunningham S.A., Kremen C., Tscharntke T. (2007) Importance of pollinators in changing landscapes for world crops. Proceedings of the Royal Society B-Biological Sciences 274:303-313.

Knauer A.C., Schiestl F.P. (2017) The effect of pollinators and herbivores on selection for floral signals: a case study in Brassica rapa. Evolutionary Ecology 31:285-304.

Krupnick G.A., Weis A.E. (1999) The effect of floral herbivory on male and female reproductive success in Isomeris arborea. Ecology 80:135-149. 
Liao K., Gituru R.W., Guo Y.H., Wang Q.F. (2013) Effects of floral herbivory on foraging behaviour of bumblebees and female reproductive success in Pedicularis gruina (Orobanchaceae). Flora-Morphology, Distribution, Functional Ecology of Plants 208:562-569.

Litto M., Scopece G., Fineschi S., Schiestl F.P., Cozzolino S. (2015) Herbivory affects male and female reproductive success differently in dioecious Silene latifolia. Entomologia Experimentalis et Applicata 157:60-67.

MacArthur R.H., Pianka E.R. (1966) On optimal use of a patchy environment. American Naturalist 100: 603-608.

MacKenzie K.E. (1994) The foraging behaviour of honey bees (Apis mellifera $L$ ) and bumble bees (Bombus spp) on cranberry (Vaccinium macrocarpon Ait). Apidologie 25:375-383.

Mallinger R.E., Gratton C. (2015) Species richness of wild bees, but not the use of managed honeybees, increases fruit set of a pollinator-dependent crop. Journal of Applied Ecology $52: 323-330$.

Mothershead K., Marquis R.J. (2000) Fitness impacts of herbivory through indirect effects on plant-pollinator interactions in Oenothera macrocarpa. Ecology 81:30-40.

Mutikainen P., Delph L.F. (1996) Effects of herbivory on male reproductive success in plants. Oikos 75:353-358.

Parachnowitsch A.L., Caruso C.M. (2008) Predispersal seed herbivores, not pollinators, exert selection on floral traits via female fitness. Ecology 89:1802-1810.

Parachnowitsch A.L., Raguso R.A., Kessler A. (2012) Phenotypic selection to increase floral scent emission, but not flower size or colour in bee-pollinated Penstemon digitalis. New Phytologist 195:667-675.

Parker J.D., Burkepile D.E., Hay M.E. (2006) Opposing effects of native and exotic herbivores on plant invasions. Science 311:1459-1461.

Potts S.G., Biesmeijer J.C., Kremen C., Neumann P., Schweiger O., Kunin W.E. (2010). Global pollinator declines: trends, impacts and drivers. Trends in Ecology \& Evolution 25:345-353.

Quesada M., Bollman K., Stephenson A.G. (1995) Leaf damage decreases pollen production and hinders pollen performance in Cucurbita texana. Ecology 76:437-443. 
513 Rader R., Howlett B.G., Cunningham S.A., Westcott D.A., Newstrom-Lloyd L.E., Walker M.K., Teulon D.A.J., Edwards W. (2009) Alternative pollinator taxa are equally efficient but not as effective as the honeybee in a mass flowering crop. Journal of Applied Ecology 46:10801087.

Rader R., Bartomeus I., Garibaldi L.A., Garratt M.P., Howlett B.G., Winfree R., et al. (2016) NonNational Academy of Sciences USA 113:146-151.

Rey P.J., Herrera C.M., Guitian J., Cerda X., Sanchez-Lafuente A.M., Medrano M., Garrido J.L. (2006) The geographic mosaic in predispersal interactions and selection on Helleborus foetidus (Ranunculaceae). Journal of Evolutionary Biology 19:21-34

Rosas-Guerrero V., Aguilar R., Marten-Rodriguez S., Ashworth L., Lopezaraiza-Mikel M., Bastida J.M., Quesada M. (2014) A quantitative review of pollination syndromes: Do floral traits predict effective pollinators? Ecology Letters 17:388-400.

Rusman Q., Lucas-Barbosa D., Poelman E.H. (2018) Dealing with mutualists and antagonists: Specificity of plant-mediated interactions between herbivores and flower visitors, and consequences for plant fitness. Functional Ecology 324:1022-1035.

Sauve A.M., Thébault E., Pocock M.J., Fontaine C. (2016) How plants connect pollination and herbivory networks and their contribution to community stability. Ecology.

Schemske D.W., Bradshaw H.D. (1999) Pollinator preference and the evolution of floral traits in monkey flowers (Mimulus). Proceedings of the National Academy of Science USA 96:11910-11915.

Schiestl F.P., Johnson S.D. (2013) Pollinator-mediated evolution of floral signals. Trends in Ecology \& Evolution 28:307-315.

Schiestl F.P., Kirk H., Bigler L., Cozzolino S., Desurmont G.A. (2014) Herbivory and floral signaling: phenotypic plasticity and tradeoffs between reproduction and indirect defense. New Phytologist 203:257-266. 
Shavit O., Dafni A., Ne'eman G. (2009) Competition between honeybees (Apis mellifera) and native solitary bees in the Mediterranean region of Israel-Implications for conservation. Israel Journal of Plant Sciences 57:171-183.

Sletvold N., Moritz K.K., Ågren J. (2015) Additive effects of pollinators and herbivores result in both conflicting and reinforcing selection on floral traits. Ecology 96:214-221.

Smallegange R.C., Van Loon J.J.A., Blatt S.E., Harvey J.A., Agerbirk N., Dicke M. (2007) Flower vs. leaf feeding by Pieris brassicae: glucosinolate-rich flower tissues are preferred and sustain higher growth rate. Journal of Chemical Ecology 33:1831-1844.

Stein K., Coulibaly D., Stenchly K., Goetze D., Porembski S., Lindner A. et al. (2017) Bee pollination increases yield quantity and quality of cash crops in Burkina Faso, West Africa. Scientific Reports 7:17691.

Strauss S.Y., Conner J.K., Rush S.L. (1996) Foliar herbivory affects floral characters and plant attractiveness to pollinators: implications for male and female plant fitness. American Naturalist 147:1098-1107.

Van Rossum F., Stiers I., Van Geert A., Triest L., Hardy O.J. (2011) Fluorescent dye particles as pollen analogues for measuring pollen dispersal in an insect-pollinated forest herb. Oecologia 165:663-674.

Ward N.L., Masters G.J. (2007) Linking climate change and species invasion: an illustration using insect herbivores. Global Change Biology 13:1605-1615.

Watanabe M., Ito A., Takada Y., Ninomiya C., Kakizaki T., Takahata Y., et al. (2000) Highly divergent sequences of the pollen self-incompatibility (S) gene in class-I S haplotypes of Brassica campestris (syn. rapa) L. FEBS Letters 473:139-144.

Winfree R., Williams N.M., Dushoff J., Kremen C. (2007) Native bees provide insurance against ongoing honey bee losses. Ecology Letters 10:1105-1113.

Winfree R., Williams N.M., Gaines H., Ascher J.S., Kremen C. (2008) Wild bee pollinators provide the majority of crop visitation across land-use gradients in New Jersey and Pennsylvania, USA. Journal of Applied Ecology 45:793-802. 
Wise M.J., Hebert J.B. (2010) Herbivores affect natural selection for floral-sex ratio in a field population of horsenettle, Solanum carolinense. Ecology 91:937-943.

\section{Figure legends}

Figure 1. Pollen staining plots: probability of pollen export (a) inside each treatment (Control for control plant, P-infested for plants infested by Pieris brassicae, M-infested for plants infested by Mamestra brassicae, and S-infested for plants infested by Spodoptera littoralis), or (b) from a given plant treatment to any other plant treatment. Each pollen movement combination is designated by two letters: the first indicates the pollen source, the second the pollen receiver $(C=$ control plants, $\mathrm{P}=$ plants infested by $P$. brassicae, $\mathrm{M}=$ plants infested by $M$. brassicae; $\mathrm{S}=$ plants infested by $S$. littoralis). (c) Pollen dispersal distance of stained-pollen from each treatment.

Figure 2. Correlation between the number of open flowers and the number of visits for (a) honey bees and (b) other wild pollinators. (c) Number of visits of honey bees (black) and other wild pollinators (grey) to plants exposed to different treatments. Non-significant values are indicated in light grey and significant values in black. ${ }^{*} P<0.05,{ }^{* *} P<0.01$.

Figure 3. Pollinator choices facing different herbivory treatments. (a) Total number of visits by honey bees (left panel) and other wild pollinators (right panel) to the three different treatments (Control plants; P-infested: plants infested by Pieris brassicae; S-infested: plants infested by Spodoptera littoralis); (b) Number of honey bees (left panel) and other wild pollinators (right panel) visiting the three different plant treatments as first choice. (c) Pollinators shifts among plants exposed to different treatments for both categories of pollinators (honey bees on left panel and 
594 other wild pollinators on right panel). Each pollen movement combination is designated by two 595 letters: the first indicates the pollen source, the second the pollen receiver $(C=$ control plants, $P=$ 596 plants infested by $P$. brassicae, $\mathrm{M}$ = plants infested by $M$. brassicae; $\mathrm{S}=$ plants infested by $S$. 597 littoralis).

\section{Supporting information}

600 Table S1. Date, time of exposure to pollinators and total number of dye movements for the seven 601 pollen staining plots.

602

Figure S1. Comparison of herbivore damage in plants infested by the wild generalist Mamestra brassicae, the wild specialised Pieris brassicae and the invasive generalist Spodoptera littoralis. 605

Figure S2. Pollinators observed in the experimental plots and their relative abundance.

Figure S3. Comparison of floral traits (a) Petal width, (b) Petal length, (c) Sepal width (d) Sepal length, among plants exposed to different treatments (Control plants, P-infested: plants infested by Pieris brassicae, S-infested: plants infested by Spodoptera littoralis).

Figure S4. Comparison of plant height (a) and number of flowers (b) for the different herbivore 613 treatments. 

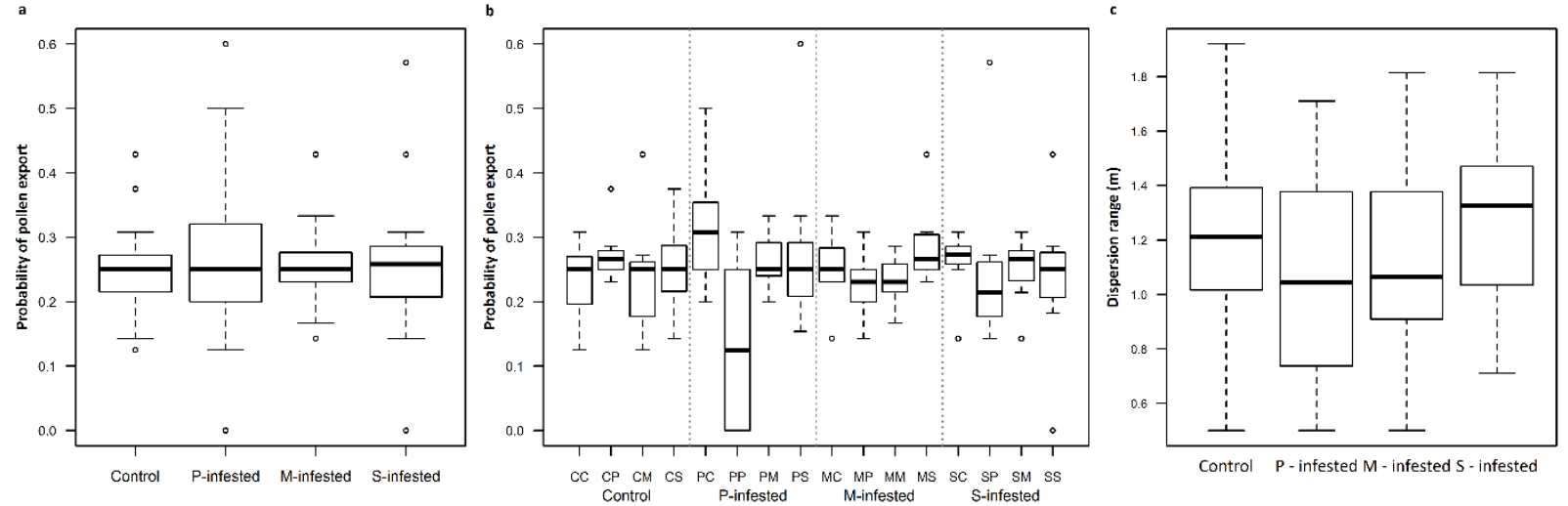

Figure 2
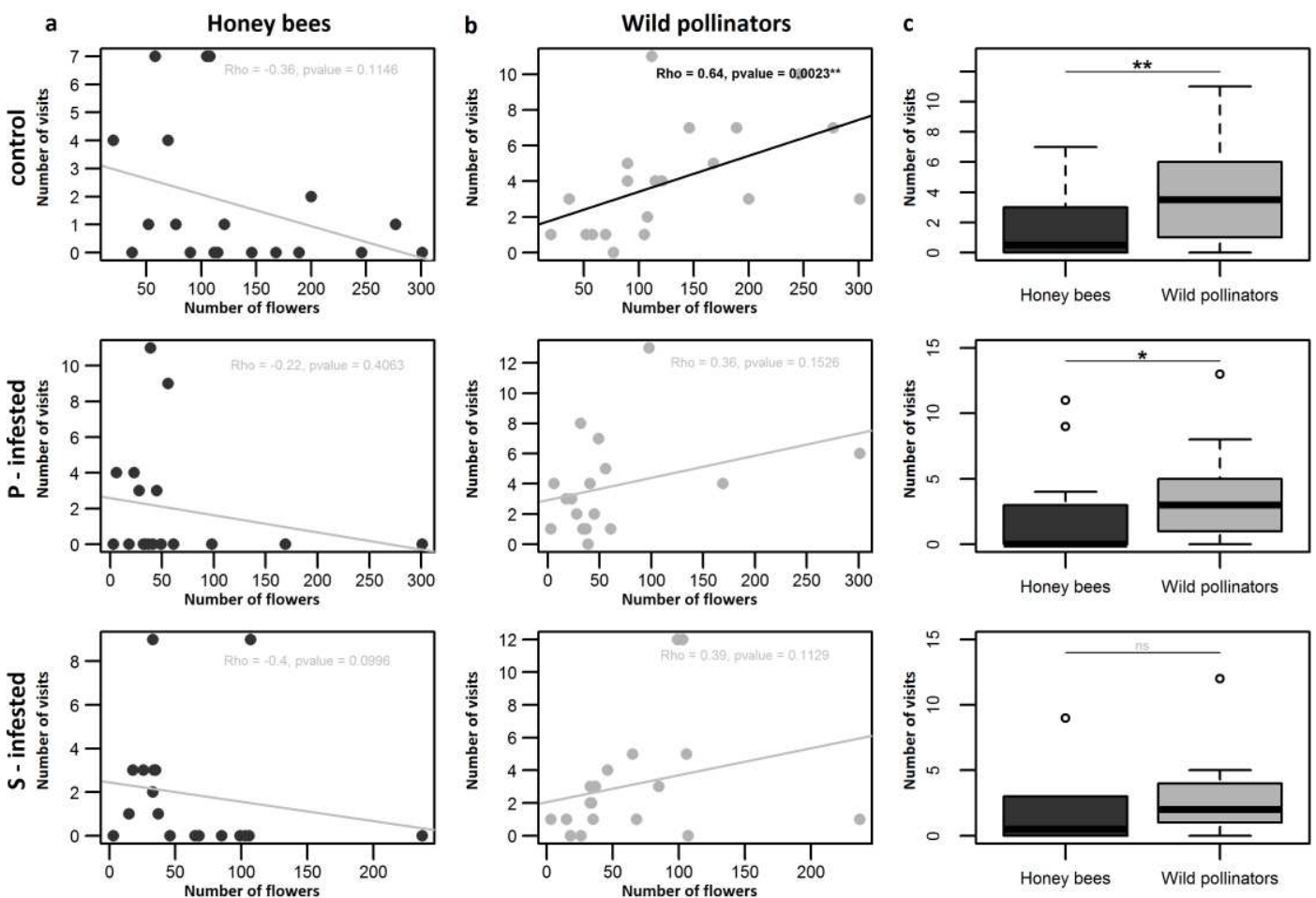
652 Figure 3

653

a Visit preference

Honey bees
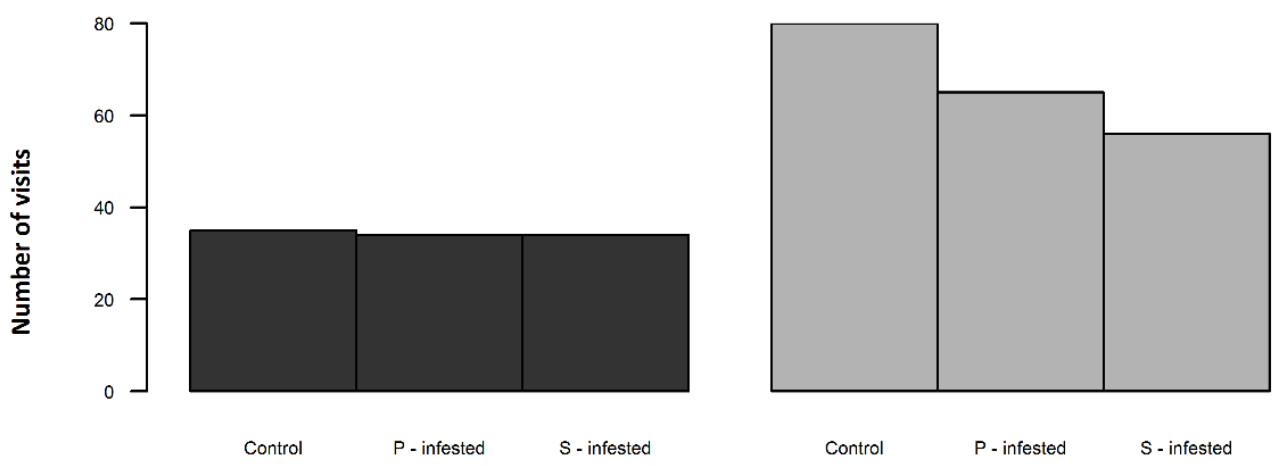

Control $\quad P$-infested $\quad S$-infested

b $\quad$ First visit
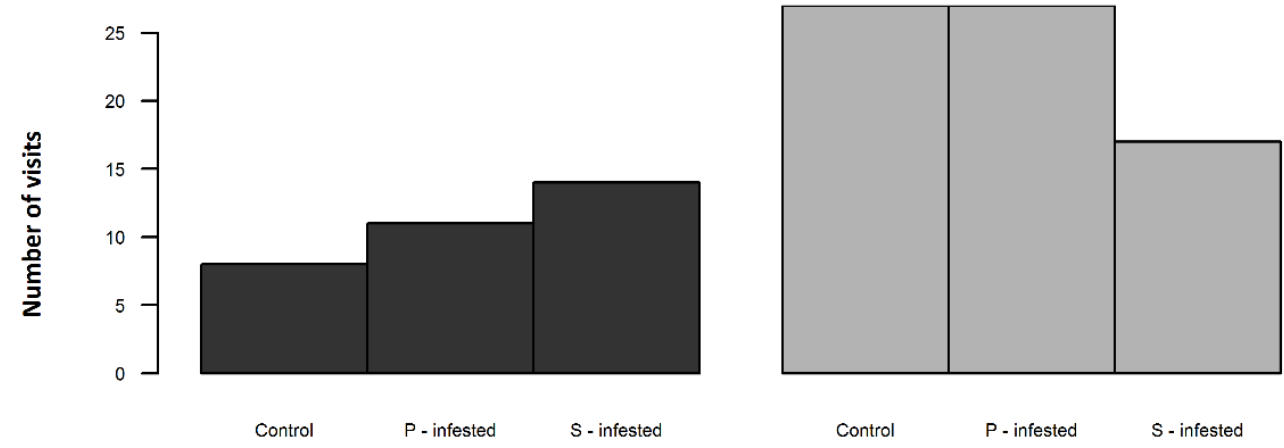

Control $\quad P$-infested $\quad S$-infested

C Pair of visits

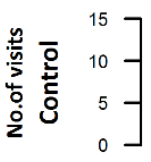

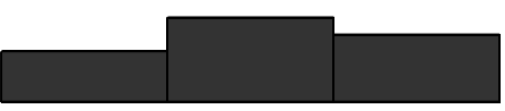

$\mathrm{CC}$

$\mathrm{CP}$

CS

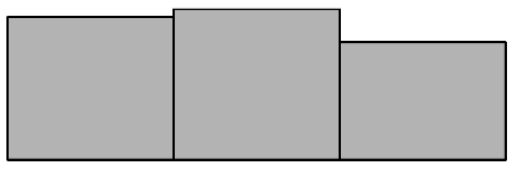

cC

$\mathrm{CP}$

cs

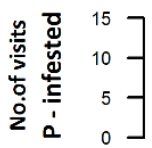

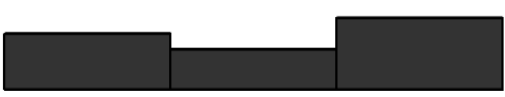

PC

PP

PS

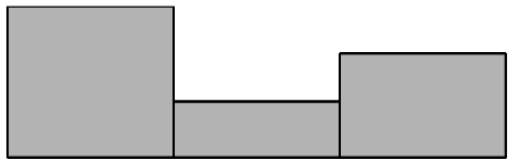

PC

PS

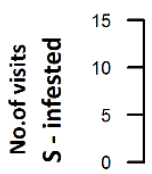

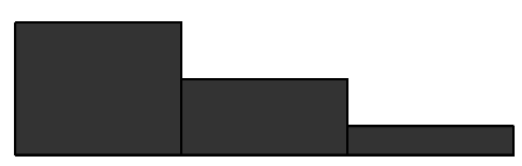

SC

SP

SS

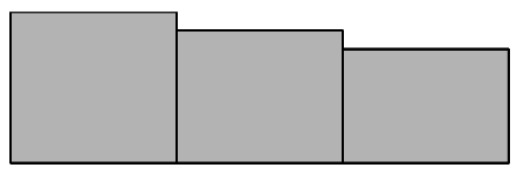

SC

SP

ss 